method, the adjoint equations are homogeneous and the nonhomogeneity is postponed to the algorithm (9) for obtaining the corrections. As to which method is faster or more efficient, it is not possible at this time to say. Some time later Brown [3], in connection with a problem in the theory of hydrodynamic stability, independently introduced a method using initial value problems, but convergence to the solution was achieved partially by trial and error so that the method is not fully automatic. This objection was overcome by Nachtsheim [4] who used a perturbation scheme and iterated to the final solution; he was compelled, however, to estimate more constants than are truly required. Although all of these investigators worked on the same problem, none of them seems to have been aware of his predecessors.

The principles of the method presented here may be applied to solve nonlinear eigenvalue problems, since, in solving initial value problems, the computer is indifferent to linearity. Of course, the equations of differential corrections are linear in any case, and so are their adjoints; but in this case the coefficients depend on the previous iteration of the eigenfunctions. In nonlinear cases the solution cannot be arbitrarily normalized because, in contrast to the linear case, the eigenvalues depend on the amplitude of the eigenfunctions (e.g., in determining the period of a cubic spring). In fact, for nonlinear cases, the eigenvalue problem would have to be solved many times in order to grasp this dependence, and the relation between the initial value of $y_{r+1}$ and the amplitude would have to be established from the eigenfunctions. It might be pointed out that nonlinear eigenvalue problems cannot be solved using methods involving a secular equation (except for periodic solutions when one frequency dominates, in which case the method of equivalent linearization can be used as an approximation), and, in this respect, the present method is superior.

Oceanics, Inc.

Plainview, L. I., New York

1. T. R. Goodman \& G. N. LAnce, "The numerical integration of two-point boundary value problems," $M T A C$, v. 10, 1956, pp. 82-86. MR 18, 420.

2. L. Fox, The Numerical Solution of Two-Point Boundary Value Problems in Ordinary Differential Equations, Oxford Univ. Press, New York, 1957; p. 245. MR 21 * 972.

3. W. B. Brown, "Exact solution of the stability equations for laminar boundary layers in compressible flow," Boundary Layer and Flow Control: Its Principles and A pplication, Vol. 2, G. V. Lachmann, Ed., Pergamon Press, New York, 1961; pp. 1023-1038. MR 28 * 1843.

4. P. R. Nachtsheim, An Initial Value Method for the Numerical Treatment of the OrrSommerfeld Equation for the Case of Plane Poiseuille Flow, NASA TN D-2414, August, 1964.

\title{
A Special Technique For The Determination of Eigenvalues
}

\author{
By V. O. S. Olunloyo
}

1. Introduction. We consider the problem of determining an eigenvalue of pre-scribed order of the system

$$
y^{\prime \prime}+[\sigma(x)+\lambda] y=0, \quad y(0)=y(1)=0, \quad \sigma>0 .
$$

We specifically wish to avoid the eigenvalues of lower order. We may begin with a reasoned guess based partly on classical inequalities. The problem then boils down

Received July 6, 1964. Revised November 10, 1964. 
to the determination of the eigenvalue nearest a given number, a situation which was studied by Kryloff and Bogoliuboff [1]. One deals essentially with intervals containing the nearest eigenvalue as an interior point, the main task being to reduce these intervals as much as possible. When the guessed value is nearer another eigenvalue other than the specific one sought, there is, in general, a tendency to drift towards the nearest eigenvalue. It is shown here how this drift may be checked. In this paper, the theory of Kryloff and Bogoliuboff is examined. An error is pointed out and the theory is reduced to a numerical method. An interesting computational example involving Mathieu functions is constructed and treated.

2. On the Theory of Kryloff and Bogoliuboff. Details would be found in [1]. We consider the minimization of

$$
J_{k}(\zeta)=\int_{0}^{1}\left[\zeta^{\prime \prime}+(\sigma+k) \zeta\right]^{2} d x / \int_{0}^{1} \zeta^{2} d x
$$

$\zeta$ is expanded in a Fourier trigonometric series of the exact eigenfunctions $y_{i}$.

$$
\zeta(x)=\sum_{i=1}^{\infty} h_{i} y_{i}, \quad h_{i}=\int_{0}^{1} \zeta(x) y_{i} d x .
$$

On assuming the series is twice differentiable, one gets

$$
J_{k}(\zeta)=\sum_{i=1}^{\infty} h_{i}{ }^{2}\left(\lambda_{i}-k\right)^{2} / \sum_{i=1}^{\infty} h_{i}{ }^{2}
$$

where the eigenvalue nearest $k$ is $\lambda_{j}$, say. The co-ordinate functions employed,

$$
\psi_{i}=\sqrt{ } 2 \operatorname{Sin} \pi i x, \quad \zeta_{m}=\sum_{i=1}^{m} A_{i} \psi_{i},
$$

lead to

$$
\int_{0}^{1}\left[E_{k}\left(\zeta_{m}\right) E_{k}\left(\psi_{i}\right)-\nu \zeta_{m} \psi_{i}\right] d x=0 \quad(i=1,2, \cdots, m),
$$

where $\nu$ is a Lagrange multiplier to be determined and

$$
\nu=d_{m}=J\left(\zeta_{m}\right) \text {. }
$$

A string of inequalities based largely on Parseval's relation and the Cauchy-Schwarz inequality lead eventually to

$$
0 \leqq d_{m}-\left(\lambda_{j}-k\right)^{2}<\eta_{m},
$$

where

$$
\begin{aligned}
\eta_{m} & =\frac{\left[1+\frac{|k \widehat{+} \sigma|^{2}}{(m+1)^{2} \pi^{2}}\right] \frac{A^{2}}{(m+1)^{4} \pi^{4}}}{1-\frac{A^{2}}{(m+1)^{8} \pi^{8}}} \\
A & =\left\{\left|\left(\lambda_{j}+\sigma\right)^{2}-\sigma^{\prime \prime}\right|-2\left|\sigma^{\prime} \sqrt{ }\left(\left|\lambda_{j}\right|+|\sigma|\right)\right|\right\}
\end{aligned}
$$

and

$$
\lambda=k \pm \sqrt{ }\left(d_{m}-\theta \eta_{m}\right), \quad 0<\theta<1
$$


If $d_{m}<\eta_{m}$ we can conclude that $\lambda_{j} \leqq k+\sqrt{ } \eta_{m}$ but, if $d_{m}>\eta_{m}$, we get two intervals,

$$
\text { (I) } k \pm \sqrt{ }\left(d_{m}-\eta_{m}\right), \quad \text { (II) } k \pm \sqrt{ } d_{m} \text {. }
$$

In the first, no real eigenvalue exists and, in the other, there is at least one. The neighbouring eigenvalues may then be isolated with $m$ sufficiently large.

As $m \rightarrow \infty$, (I) and (II) approach and

$$
\sqrt{ } d_{m}-\sqrt{ }\left(d_{m}-\eta_{m}\right)<\sqrt{ } \eta_{m}
$$

The vital intervals are, therefore,

$$
\left(k-\sqrt{ } d_{m}, k-\sqrt{ }\left(d_{m}-\eta_{m}\right)\right) \text {, and }\left(k+\sqrt{ } d_{m}, k+\sqrt{ }\left(d_{m}-\eta_{m}\right)\right) .
$$

The determination of the sign of the correction in [1] may, in certain circumstances, be replaced by the suggestions which follow.

3. Reduction of the Theory to a Numerical Method. We now examine how to decrease an interval containing an eigenvalue. We note that $\eta_{m}$ is not explicitly known, since $A$ is unknown, and has to be estimated. An upper estimate, while logically safe, is, of course, not necessarily 'best'. When $\sigma>0$ and $\sigma^{\prime}<0$, as, for example, in problems involving Mathieu functions, the case is clear. In any case,

$$
A \leqq\left|\left(\lambda_{j}+\sigma\right)^{2}\right|+\left|\sigma^{\prime \prime}\right|+2\left|\sigma^{\prime} \sqrt{ }\left(\left|\lambda_{j}\right|+|\sigma|\right)\right| \text {. }
$$

Thus $\eta_{m}$ increases with $A$. We consider now in detail the choice between the two vital intervals. There is a criterion that settles where $\lambda_{j}$ is. In the derivation of this criterion, however, an error occurs in [1]. Let the end parts of interval (I) be $k_{1}, k_{2}$ and the eigenvalue nearest $t$ be $\lambda_{t}$, say. One may deduce

$$
\begin{aligned}
& 0 \leqq d_{1, m}-\left(\lambda_{k_{1}}-k_{1}\right)^{2}<\bar{\eta}_{m}, \\
& 0 \leqq d_{2, m}-\left(\lambda_{k_{2}}-k_{2}\right)^{2}<\bar{\eta}_{m},
\end{aligned}
$$

where

$$
\bar{\eta}_{m}=\frac{\left[1+\frac{|k|+|\sigma|+\left|\sqrt{ } d_{m}\right|}{(m+1)^{2} \pi^{2}}\right] \frac{A^{2}}{(m+1)^{4} \pi^{4}}}{1-\frac{A^{2}}{(m+1)^{8} \pi^{8}}}
$$

$k$ has been replaced by the upper bound $k+\sqrt{ } d_{m} . d_{1, m}$ and $d_{2, m}$ are obtained from $k_{1}, k_{2}$, respectively, by repeating previous routines. Separate consideration of the cases $k<\lambda_{j}, k>\lambda_{j}$ is necessary.

Case A. If $k<\lambda_{j}$, then $k+\sqrt{ } d_{m} \geqq \lambda_{j} \geqq k_{1}$,

$$
0 \leqq \lambda_{j}-k_{1} \leqq \sqrt{ } d_{m}-\sqrt{ }\left(d_{m}-\eta_{m}\right)
$$

In [1] it is stated that

$$
\sqrt{ } d_{m}-\sqrt{ }\left(d_{m}-\eta_{m}\right)<\frac{\eta_{m}}{2 \sqrt{ } d_{m}} .
$$

This is false because it implies

$$
1-\sqrt{ }(1-u)<\frac{u}{2}, \quad u=\frac{\eta_{m}}{d_{m}}<1,
$$


i.e., $0 \leqq 1-u / 2<\sqrt{ }(1-u)$ and $1-u+u^{2} / 4<1-u$ and $u^{2}<0$ for real $u$. Moreover, this error is carried forward in the derivation of two inequalities for $d_{1, m}$ and $d_{2, m}$. In fact,

$$
\sqrt{ } d_{m}-\sqrt{ }\left(d_{m}-\eta_{m}\right)<\sqrt{ } \eta_{m}
$$

therefore,

$$
0 \leqq\left(\lambda_{j}-k_{1}\right) \leqq \sqrt{ } \eta_{m} .
$$

It is clear that, in the case $k<\lambda_{j}, \lambda_{k_{1}}=\lambda_{j}$,

$$
d_{1, m}<\left(\lambda_{k_{1}}-k_{1}\right)^{2}+\bar{\eta}_{m} \leqq \bar{\eta}_{m}+\eta_{m}=\epsilon_{m} .
$$

Case B. If $k>\lambda_{j}$, one sees similarly that $d_{2, m} \leqq \epsilon_{m}$. If $k \neq \lambda_{j}$, either Case A or Case B will hold. We thus have a criterion for deciding which vital interval to choose. Clearly, if $d_{1, m}>\epsilon_{m}$ then $\lambda_{j}<k$ and if $d_{2, m}>\epsilon_{m}$ then $\lambda_{j}>k$. Thus, starting with a specific value of $k$, one may calculate $d_{m}, \eta_{m}$ and hence $k_{1}, k_{2}$. One can then find $d_{1, m}$ and $d_{2, m}$ and also $\epsilon_{m}$. Upper bounds being needed for $\eta_{m}$ and $\epsilon_{m}$, one has now two vital intervals. The sign of $d_{1, m}-\epsilon_{m}$ or $d_{2, m}-\epsilon_{m}$ is determined. Whichever is negative belongs to the relevant vital interval. The subsequent systematic reduction of that interval may be achieved as follows. Suppose the left-hand interval is the vital one; one calculates a value of $\sqrt{ }\left(d_{m}-\eta_{m}\right)$ to define $k_{3}$, etc., until, however, $d_{m}<\eta_{m}$. This, therefore, establishes the feasibility of a practical use of the theory in [1]. Drift towards an unwanted eigenvalue can now be checked. If one made an upper estimate that was too high (or a lower one, too low) one would tend to drift towards the next higher (or lower) eigenvalue. This drift can be detected when $d_{1, m}$ and $d_{2, m}$ are calculated. It can be rectified by reducing $k$ somewhat arbitrarily until the left-hand interval becomes relevant. Care must, of course, be exercised to ensure that the reduction is not so drastic as to create a lower bound that is too small.

4. A Numerical Example. We seek only the fifth eigenvalue of the system

$$
\begin{aligned}
& y^{\prime \prime}+\left(\lambda+200 \operatorname{Sin}^{2} \pi x\right) y=0=y(0)=y(1), \\
& \sigma(x)=200 \operatorname{Sin}^{2} \pi x, \quad \sigma^{\wedge}=200 \text { and } \sigma^{\vee}=0 .
\end{aligned}
$$

By Sturm's comparison theorem [2],

$$
\begin{aligned}
\lambda_{5}+200 & \geqq(5 \pi)^{2} \geqq \lambda_{5}, \\
46.74 & \leqq \lambda_{5} \leqq 246.74 .
\end{aligned}
$$

The weakness of this inequality shows the nontriviality of the chosen example. One may quickly obtain an upper bound by the simplest variation method, viz., Rayleigh principle. We choose $y=$ Sin $5 \pi x$ and get $\lambda_{5}<147$. Thus

$$
46<\lambda_{5}<147 \text {. }
$$

Start with the convenient guess $k=100$. The equations (6) are set up with

$$
\begin{aligned}
\zeta_{m} & =\sum_{p=1}^{m} a_{p}(\sqrt{ } 2 \operatorname{Sin} p \pi x), \\
E_{k}\left(\zeta_{m}\right) & =\sum_{p=1}^{m}\left[k-(p \pi)^{2}+200 \operatorname{Sin}^{2} \pi x\right] a_{p} \sqrt{ } 2 \operatorname{Sin} p \pi x
\end{aligned}
$$


The cases $p=1,2,3$ and 4 need special care.

$$
\begin{gathered}
\left(c_{1}^{2}+100 c_{1}+5000-\nu\right) a_{1}+\left(-50 c_{1}-50 c_{3}-2500\right) a_{3}+2500 a_{5}=0 \\
\left(c_{2}^{2}+2500-\nu\right) a_{2}+\left(-50 c_{2}-50 c_{4}\right) a_{4}+2500 a_{6}=0 \\
\left(-50 c_{3}-50 c_{1}-2500\right) a_{1}+\left(c_{3}^{2}+5000-\nu\right) a_{3} \\
+\left(-50 c_{3}-50 c_{5}\right) a_{5}+2500 a_{7}=0 \\
\left(-50 c_{2}-50 c_{4}\right) a_{2}+\left(c_{4}^{2}+5000-\nu\right) a_{4} \\
+\left(-50 c_{4}-50 c_{6}\right) a_{6}+2500 a_{8}=0 .
\end{gathered}
$$

For $m-4 \geqq i \geqq 5$,

$$
\begin{aligned}
2500 a_{i-4}+\left(-50 c_{i-2}-50 c_{i}\right) a_{i-2} & +\left(5000+c_{i}^{2}-\nu\right) a_{i} \\
+ & \left(-50 c_{i}-50 c_{i+2}\right) a_{i+2}+2500 a_{i+4}=0,
\end{aligned}
$$

with

$$
a_{t}=0, \quad t>m, \quad c_{\mathbf{\imath}}=k+100-(i \pi)^{2} .
$$

The lowest latent root is then extracted and $A$ and $\eta_{m}$ are calculated. The calculation was based on a $20 \times 20$ matrix.

$$
A(\lambda)=(\bar{\lambda}+200)^{2}+400 \pi^{2}+400 \pi \sqrt{ }(\lambda+200),
$$

where $\bar{\lambda}$ denotes an upper bound to $\lambda_{j}$.

$$
A(147)=347^{2}+400 \pi^{2}+400 \pi \sqrt{ } 347=146766 .
$$

With $m=20, k=100$ we have $\eta_{m}=13170$. Also, $d_{m}$ was found to be 14073.75 . Here $d_{m}>\eta_{m}, d_{m}-\eta_{m}=903.75, \sqrt{ } d_{m}=118.63, \sqrt{ }\left(d_{m}-\eta_{m}\right)=30.062$. Thus we have the interesting result that $\lambda_{5}$ lies either in $(-18,70)$ or $(130,219)$. But as $46<\lambda_{5}<147$, we see that either $\lambda_{5}$ lies in

$$
(46,70) \text { or }(130,147) \text {, }
$$

which are much narrower intervals. To determine actually which is the relevant vita $l$ interval, we calculate $d_{1,20}$ and $d_{2,20}$ by previous routine for $k_{1}$ and $k_{2}$. A certain amount of flexibility may be very useful here. One may choose to calculate $d$ for two other values of $k$ instead of 69.938 and 130.06. This involves an identical amount of labour. We could consider mean points, viz., 58 and 138. In addition to providing estimates in their own right, they should be combined with the previous results. By taking arithmetic means systematically one may succeed in accelerating convergence. The purpose of the illustration can thus be regarded as fulfilled. The exact solution can be expressed as in [3] as a Mathieu function of an irrational order and $\lambda_{5}$ is slightly over 55 . Incidentally, $\lambda_{6}$ is $164 \pm 1$ and $\lambda_{4}$, which is negative, is about -34 . The exact values could have been interpolated between table values for rational order Mathieu functions but the figures quoted are within a whole number of the exact result. The idea of trying 58 and 138, in particular, and also of the value of a 
numerical method based on the theory of Kryloff and Bogoliuboff will now be apparent.

University of Ife

Ibadan, Nigeria p. 471 .

1. N. M. Kryloff \& N. Bogoliuboff, Izv. Akad. Nauk S.S.S.R. Ser. Mat. Fiz., 1929,

2. J. C. Burkill, The Theory of Ordinary Differential Equations, University Mathematics Texts, Oliver and Boyd, Edinburgh, 1956.

3. N. W. McLachlan, Theory and Application of Mathieu Functions, Clarendon Press; Oxford, 1947. MR 9, 31.

\section{Integration Rules of Hypercubic Symmetry over a Certain Spherically Symmetric Space}

\section{By J. N. Lyness}

Abstract. A theory of integration rules suitable for integration over a hypercube and having hypercubic symmetry has recently been published. In this paper it is found that, with minor modification, this theory may be directly applied to obtain integration rules of hypercubic symmetry suitable for integration over a complete $n$-dimensional space with the weight function $\exp \left(-x_{1}{ }^{2}-x_{2}{ }^{2} \cdots-x_{n}{ }^{2}\right)$. As in the case of integration over hypercubes, an $n$-dimensional rule of degree $2 t+1$ may be constructed requiring a number of function evaluations of order $2^{t} n^{t} / t$ !, only.

1. Introduction. In this paper we are interested in generalising the theory and results of investigations about the use of symmetric integration rules for a hypercube, given in Lyness [2] and [3] which we refer to as Part I and Part II, respectively. The particular generalisation that we consider here is the construction of rules of the type

$$
\begin{aligned}
\int_{-\infty}^{\infty} \int_{-\infty}^{\infty} \cdots \int_{-\infty}^{\infty} \exp \left[-x_{1}{ }^{2}-x_{2}{ }^{2} \cdots-x_{n}{ }^{2}\right] f\left(x_{1}, x_{2}, \cdots, x_{n}\right) d x_{1} d x_{2} \cdots d x_{n} \\
\simeq \sum A_{i} f\left(\iota_{1 i}, \mathfrak{s}_{2 i}, \cdots, \text { s } n i\right) .
\end{aligned}
$$

Such integration rules have been considered before (Stroud and Secrest [4]). It is conventional to term such a rule to be a rule of degree $d$ if the approximate equality may be replaced by an exact equality whenever $f$ is a multinomial of degree less than or equal to $d$.

Most of the results about synmetric rules for integration over hypercubes in Parts I and II may be derived in almost identical form for this integral. To avoid unnecessary repetition, we refer to Parts I and II for the details of the derivations of such results; we indicate here only the differences or modifications in these results as they occur.

Received October 12, 1964. This research was supported in part by USAF Grant No. 62-400 to the University of New South Wales. 